\title{
Microgastria-limb reduction defect syndrome
}

INSERM

\section{Source}

INSERM. (1999). Orphanet: an online rare disease and orphan drug data base.

Microgastria-limb reduction defect syndrome. ORPHA:2538

Microgastria-limb reduction defect syndrome is a rare multiple congenital anomalies syndrome characterized by congenital microgastria and a uni- or bilateral limb reduction defect, that can include absent or hypoplastic thumbs, radius, ulna and/or amelia. Association with other variable abnormalities, including intestinal malrotation, asplenia, dysplastic kidneys, hypoplastic lungs, dysplastic corpus collosum, and abnormal genitalia, has been reported. 